\title{
Urgent Tsunami Computing
}

\author{
$1^{\text {st }}$ Finn Løvholt \\ Computational Geomechanics \\ Norwegian Geotechnical Institute \\ Oslo, Norway \\ finn.lovholt@ngi.no \\ $4^{\text {th }}$ Manuela Volpe \\ Istituto Nazionale di Geofisica e \\ Vulcanologia \\ Rome, Italy \\ manuela.volpe@ingv.it
}

\author{
$2^{\text {nd }}$ Stefano Lorito \\ Istituto Nazionale di Geofisica e \\ Vulcanologia \\ Rome, Italy \\ stefano.lorito@ingv.it \\ $5^{\text {th }}$ Jacopo Selva \\ Istituto Nazionale di Geofisica e \\ Vulcanologia \\ Bologna, Italy \\ jacopo.selva@ingv.it
}

\author{
$3^{\text {rd }}$ Jorge Macías, \\ Dpto.de Análisis Matemático, \\ Estadística e Investigación Operativa y \\ Matemática Aplicada \\ University of Malaga, Málaga, Spain \\ jmacias@uma.es
}

$6^{\text {th }}$ Steven J. Gibbons

Risk and Climate adaptation

Norwegian Geotechnical Institute

Oslo, Norway

steven.gibbons@ngi.no

\begin{abstract}
Tsunamis pose a hazard that may strike a coastal population within a short amount of time. To effectively forecast and warn for tsunamis, extremely fast simulations are needed. However, until recently such urgent tsunami simulations have been infeasible in the context of early warning and even for highresolution rapid post-event assessment. The implementation of efficient tsunami numerical codes using Graphical Processing Units (GPUs) has now allowed much faster simulations, which have opened a new avenue for carrying out simulations Faster Than Real Time (FTRT). This paper discusses the need for urgent computing in computational tsunami science, and presents workflows for two applications, namely FTRT itself and Probabilistic Tsunami Forecasting (PTF). PTF relies on a very high number of FTRT simulations addressing forecasting uncertainty, whose full quantification will be made more and more at reach with the advent of exascale computing resources.
\end{abstract}

Keywords - tsunamis, urgent computing, GPUs, probabilistic forecasting, uncertainties, workflows

\section{INTRODUCTION}

Tsunamis are long water waves that are usually triggered by large geophysical events, most often earthquakes, but also landslides or volcanoes. They can also be induced by unusual weather phenomena, in which case they are termed meteotsunamis. Tsunamis propagate over the ocean and cause damage at transoceanic distances. However, the largest tsunami damage normally occurs relatively close to its origin, where the tsunami can strike within less than an hour, sometimes even within only a few minutes. This leaves very little time for evacuating the coastal population, illuminating the demanding conditions for Tsunami Early Warning Systems (TEWS) to be effective. It is not possible at present, to predict the location, time and size of the next earthquake. Moreover, just after an earthquake has occurred, data are typically insufficient to univocally characterize the ensuing tsunami, hence the forecast needs to be provided in a regime of high uncertainty.

Tsunamis happen infrequently and, according to the NCEI tsunami database [1], 78 tsunamis have occurred between 2014 and 2018 on a global basis, which average to about 15 tsunamis per year. Of these, two recent tsunami events (Palu and Anak Krakatoa in 2018) both largely of non-seismic origin resulted in most of the fatalities, with most of the remaining events causing far fewer fatalities. However, further back in history, two major events such as the 2004 Indian Ocean and the 2011 Tohoku seismic tsunamis contributed to a major portion of the losses from all tsunamis occurring in the last 100 years.

The reasons why the greatest damage results from just a limited proportion of recorded tsunamis are manifold. First, many earthquakes produce relatively small tsunamis, either due to their relatively small magnitude (smaller earthquakes being far more frequent than the largest ones), or due to the focal mechanism not being strongly tsunamigenic. The variability in earthquake focal mechanisms results in a corresponding variability in the size and impact of the associated tsunamis, even given earthquakes with the same moment magnitude. Second, tsunami early warning systems and the population awareness of tsunami hazards have gradually improved since the 2004 Indian Ocean tsunami [2][3], in part due to improved TEWS capabilities in more and more countries [4] and in part due to the ability to estimate the earthquake moment magnitudes more rapidly and more accurately [5].

The large variability of tsunami characteristics and the need to quantify the tsunami size rapidly define important challenges with respect to urgent computing for improving present-day TEWS operations and for high-resolution rapid post event analysis tools:

- Computing the tsunami propagation Faster than Real Time (FTRT) just after the tsunami has been generated;

- Using a large number of FTRT simulations, which is critical to quickly quantify the uncertainty in the tsunami impact using all available evidence. This necessitates using a Probabilistic Tsunami Forecasting (PTF) approach, to rapidly characterize the complex tsunami propagation both over the ocean and near the source;

- Possibly updating the PTF continuously as more data becomes available, necessitating additional FTRT simulations;

This work is partially funded by the European Union's Horizon 2020 Research and Innovation Program under grant agreement No 823844 (ChEESE Center of Excellence, www.cheese-coe.eu). 
- Increasing the resolution of local tsunami inundation simulating and mapping for rapid tsunami post-event analysis and management.

To deal with these challenges, the following main elements are needed:

1) Benchmarked numerical models that can perform accurate FTRT tsunami simulations both locally and over transoceanic distances.

2) Ability to simulate many (e.g. several tens of thousands) of alternative tsunami scenarios to characterize the tsunami uncertainty in PTF within a short amount of time.

3) Efficient workflows for both FTRT and PTF.

4) Implementation of the workflows and numerical models in a relevant supercomputing environment, with significant and readily enough available computational resources.

Here, we propose urgent computing workflows for tsunami simulations, both with respect to FTRT calculations and PTF. Some brief details of the suggested workflows are presented in this paper. We stress that the workflows are under development, and the versions presented in this paper are preliminary. The method for PTF itself is under consideration for publication elsewhere.

As will be elaborated below, our example necessitates Graphical Processing Unit (GPU) clusters. Numerous independent simulations will be launched in a tier-0 PRACE HPC system using GPUs. This work is being accomplished in the framework of the EU ChEESE Center of Excellence for Exascale in Solid Earth. The associated challenges related to this work, i.e. exploring fully the uncertainties related to a high number of possible alternative high-resolution tsunami simulations, will be made more and more possible when the exascale wall $\left(\sim 10^{18}\right.$ FLOPS) is broken.

\section{Use Of URgent TsunAmi Simulations}

\section{A. Faster than Real Time (FTRT) tsunami time domain simulation}

FTRT tsunami computations are crucial in the context of TEWS and in the context of rapid post-disaster management. Greatly improved and highly efficient computational methods are the first raw ingredient for achieving extremely fast and effective calculations. HPC facilities have the role of maximizing this efficiency and drastically reducing computational times. While earthquakes cause about $80 \%$ of all tsunamis, other causes such as landslides and volcanoes, and to an extent meteotsunamis, are important as well.

Earthquake tsunami generation is in a sense simpler than landslide tsunami generation, as landslide generated tsunamis depend on the landslide dynamics which necessitate coupling dynamic landslide simulation models to the tsunami propagation. In the present context, motivated by TEWS applications, earthquakes are also more relevant as their location and magnitude can be quickly determined and used as input to FTRT modelling. To initiate simulations, earthquake focal mechanism estimations are also necessary, and information about the focal mechanism may be available roughly 10 minutes after the event. So, possibly combined with tsunami data for constraining seismic solution ambiguities, typically available some tens of minutes after the earthquake occurrence (however depending on the location of the earthquake and wave gauge stations), FTRT modelling is a potential a tool for greatly enhancing tsunami warning operations for distant earthquake sources.

Similar technology is less developed for landslides, volcanoes, and meteotsunamis, partly because these sources are less consistent and also less well understood.

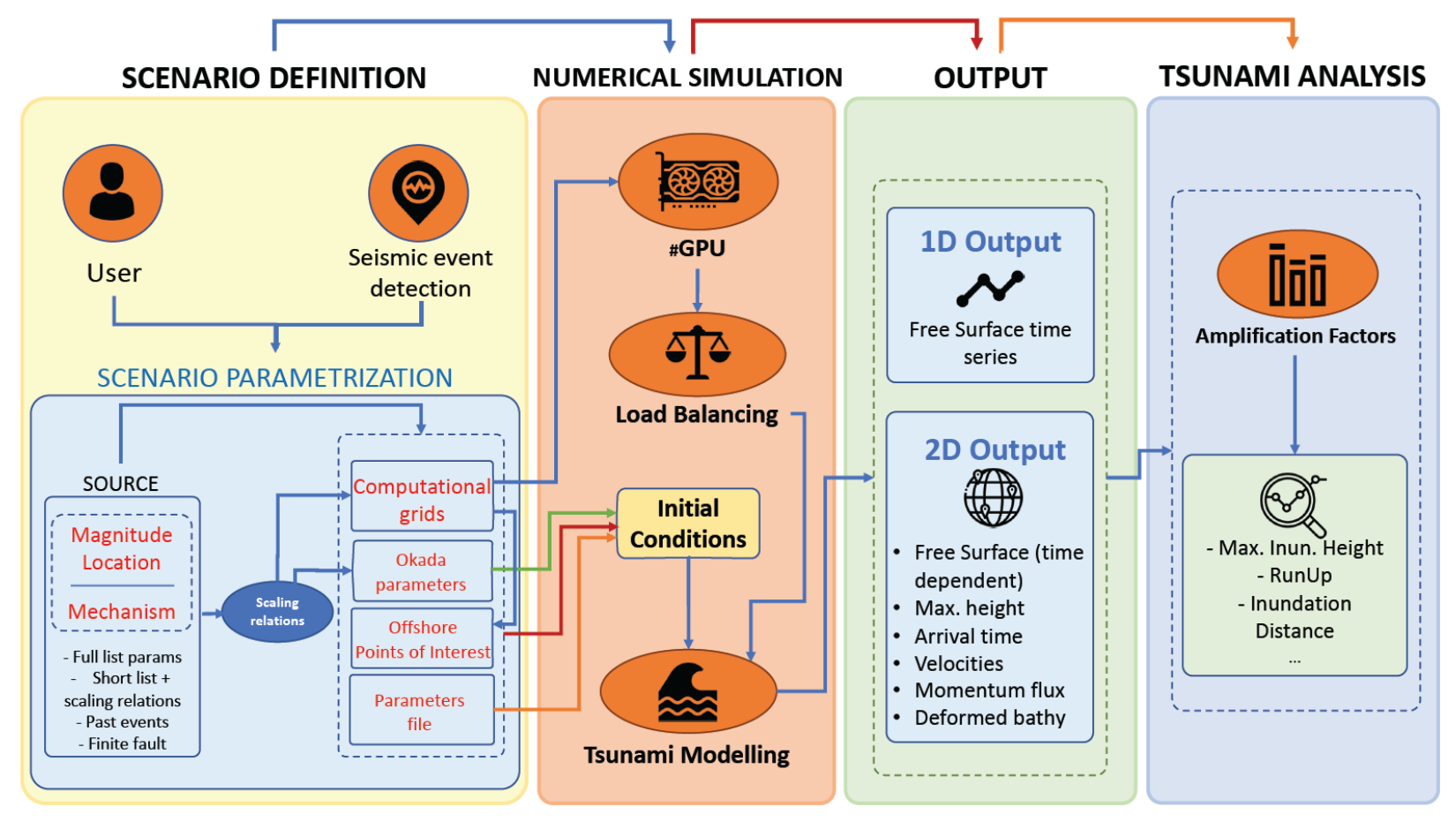

Fig. 1. Preliminary FTRT workflow. 
Tsunami simulations (see e.g. the review of Behrens and Dias [6]) normally exploit Non-Linear Shallow Water (NLSW) models, which are conducted in two horizontal dimensions (longitude and latitude) with explicit forward time-stepping. Recent NLSW models are implemented in efficient Finite Volume methods. In the models proposed here, we use the Tsunami-HySEA code [7], which is implemented using a Finite Volume scheme on GPUs. The GPU implementation renders the code supreme with respect to the possibility of carrying out simulations faster than real time.

For example, Tsunami-HySEA is presently capable of simulating the 36-hour tsunami propagation due to an earthquake over the Pacific Ocean in less than 6 minutes (using 64 NVIDIA TESLA V100 nodes on the Mare Nostrum cluster at the Barcelona Supercomputing Center) for about $84 \mathrm{M}$ horizontal nodes (2' horizontal resolution). This is indeed more than 300 times faster than the physical propagation time.

The Tsunami-HySEA code includes the option of a simple Okada type [8] model for computing the initial condition for a seismic source, but also general and more complex elastic earthquake seabed deformations from more complete models. For instance, input from 3D static or time-dependent singleand multi-segment fault geometries can be included. Moreover, the Tsunami-HySEA model also allow for filtering the sea surface response of the sea-floor displacement [9], as well as inputting the sea level displacement directly.

\section{B. Probabilistic Tsunami Forecasting (PTF)}

The available information about the seismic source immediately after the tsunami is generated is rather limited and uncertain. Hence, a relatively homogeneous single tsunami initial condition cannot be reasonably well constrained until sometime after the earthquake occurrence. Therefore, for dealing with tsunamis generated close to the target coastlines (local tsunamis) in particular, dedicated methods are needed to explore and quantify the uncertainty due to many different possible source mechanisms. This includes for instance including different candidate slip distributions and uncertainty in the source locations; both are different types of input used to the quantification of the tsunami impact uncertainty range.

A sufficiently large ensemble of FTRT simulations can be used in PTF to deal with this high uncertainty regime typical of the first minutes following the generation of a tsunami. In particular, and somehow analogous to Ensemble Prediction Systems (EPS) used for weather forecast, ensemble simulations and consequently probabilistic forecasting include both real time data, parameter, and modelling uncertainties (e.g. about the spatial size or the distribution of fault slip in a great earthquake), and their propagation into the tsunami forecast. The probability of the different potential source realizations is assigned according to their degree of consistency with the available data. The likelihood for some source parameters which are not provided soon enough by the real-time monitoring system can be estimated in a different way. The local historical and geophysical knowledge, such as the proximity to a subduction zone and local seismicity, can be used constrain the likelihood of the faulting mechanism given the location and the magnitude of the earthquake. Furthermore, stochastic simulation methods can be used for predicting earthquake slip distributions [10].

A typical case of PTF could require from thousands to hundreds of thousands of tsunami simulations for different realizations of the parameters describing the causative source. This means that the full ensemble of individual simulations would require at least the same number of GPU nodes as the number of scenarios. This also depends on the size and on the resolution of the simulation domain, and on time constraints related to the specific application. The PTF approach is still experimental and needs to be tested against past (hindcasting) and future tsunami events (forecasting). a)

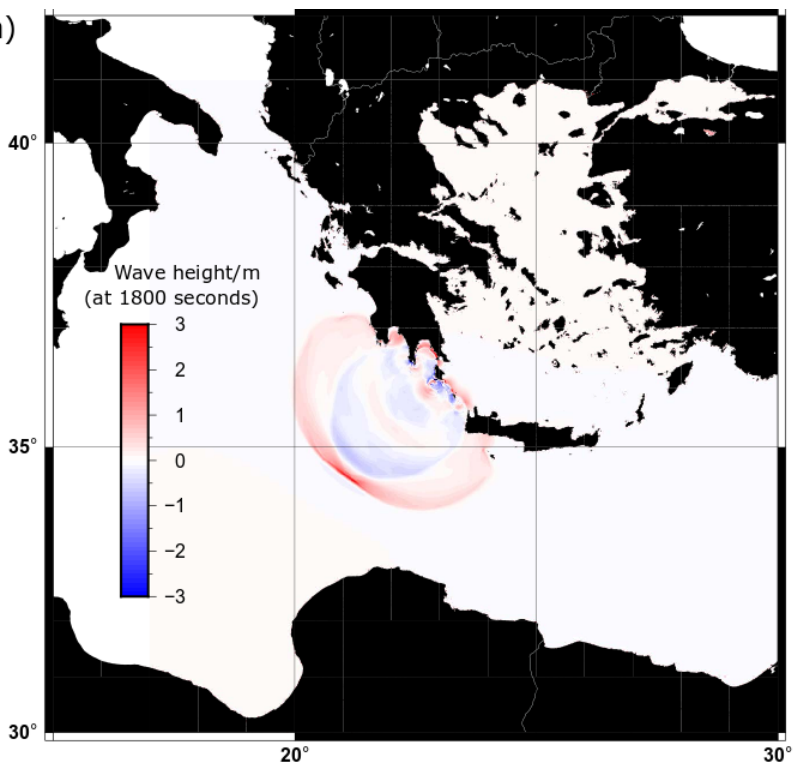

b)

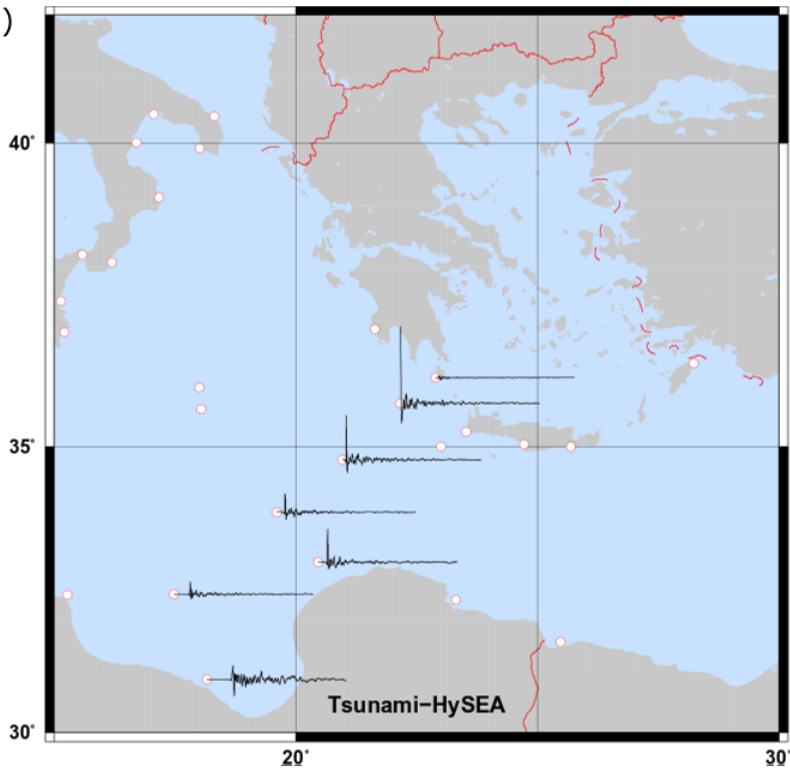

Fig. 2. Two-dimensional (a) and one-dimensional (b) output from the Tsunami-HySEA program simulating a tsunami in the Mediterranean Sea generated by a hypothetical magnitude 7.6 subduction earthquake in the Hellenic Arc. 


\section{URGENT TSUNAMI SIMULATION WORKFLOWS}

Two workflows are presented here, for the FTRT and for the PTF. The FTRT workflow is a relatively simple one and is primarily a structured way of setting up single time domain tsunami simulations and performing simple post processing. The core part of the FTRT workflow is also re-used in the PTF workflow. It is also used in a related workflow on Probabilistic Tsunami Hazard Analysis (PTHA) [11] that is outside the scope of this paper.

\section{A. Faster than Real Time (FTRT) workflow}

The FTRT workflow is outlined in Fig. 1. The first step in the workflow is the earthquake scenario parameterization. The source can either be specified manually by the user, or be triggered directly by available online data from seismic monitoring agencies, such as the earthquake focal mechanism being available very rapidly after an event has occurred. Earthquake scaling relations [12] are implemented to readily allow for immediate computation of the earthquake initial condition. Gridding tools and both public domain or proprietary high-resolution bathymetric grids can used for

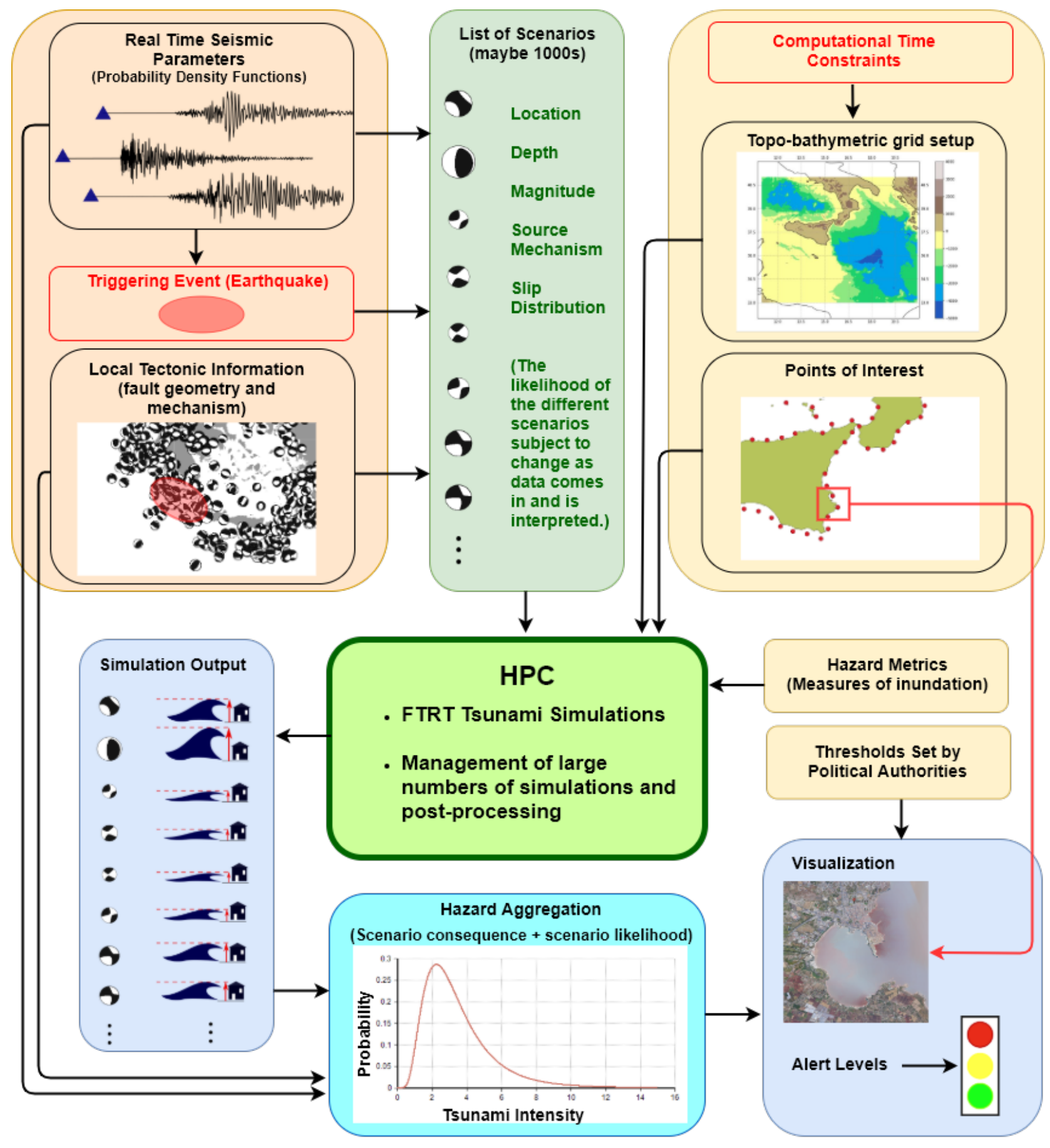

Fig. 3. Preliminary PTF workflow. 
setting up the computational meshes. For each source, threelevel grids are implemented, setting up three different domains with increasing resolution as the domain size decreases: a basin-wide domain, a regional domain and a local domain. Mesh sizes, resolution, and duration of the simulation, are based on the source information (earthquake magnitude and location). Once the source inputs and grids are available, text input files are generated, and fed as input to Tsunami-HySEA, and the numerical simulation is conducted in a GPU environment. Examples of the output from a Tsunami HySEA simulation are displayed in Fig. 2. The above facilities are reused also in the PTF workflow described below.

The FTRT workflow will further contain some simple analysis tools. These include for instance the processing of tsunami surface elevations at selected Points Of Interest (POIs) in the ocean and close to the shore, snapshots of the tsunami surface elevations at different times, arrival times, etc. The near coastal POIs are used to estimate roughly the uncertainty in the Maximum tsunami Inundation Height (MIH). Amplification factors [13]-[14] are applied for estimating onshore MIHs from tsunami offshore surface elevations calculated at the near-shore POIs in the FTRT simulations. All this information is useful for rapid post assessment of a tsunami event.

The workflow design will need to include intelligent postprocessing to ensure that only essential output from the simulations is written to file. Fig. 2 displays at left a snapshot of surface elevation for a single time point and, at right, surface elevation time-series for a small number of selected locations. It is from such time-series at near-shore POIs that the polarity, maximum amplitude, and period are calculated to estimate the statistics of the likely on-shore inundation using the amplification factor method [13]-[14]. Writing out frequent full-domain snapshots of variables puts pressure both on I/O and storage resources. On the other hand, quantities such as wave arrival time and maximum elevation height attained have only a single value for each grid location of the full-domain for a given simulation and can be output without overwhelming resources.

\section{B. Probabilistic Tsunami Forecasting (PTF) workflow}

The PTF workflow is outlined in Fig. 3. A prototype implementation of the PTF workflow for the Mediterranean Sea already exists and it is currently being tested at INGV with a view towards operational deployment. This prototype version is based on a database of pre-calculated tsunami scenarios. In the new ChEESE workflow presented in this paper, the database will be replaced by FTRT tsunami simulations for a list of scenarios, and applicable in principle to any possible event worldwide.

Real-time seismic parameters are estimated by the EARLY-EST software running at the CAT-INGV Tsunami Warning Centre [15]. The computational procedure will be also supplemented by seismic parameters from other agencies; different estimates will be treated as an ensemble. The local tectonic information as shown in Fig. 3 indicates use of static (as based on past data) information for seismic parameters typically unavailable in the very first minutes (for example the earthquake focal mechanism). After the triggering event information is available, these static conditional probabilities are updated conditioned by new information. All conditional probabilities, as well as all precomputed earthquake scenarios, are taken from the
TSUMAPS-NEAM PTHA project results (http://www.tsumaps-neam.eu/). As noted above, we will replace these precomputed scenarios in the new ChEESE prototype with large ensembles of FTRT simulations.

Due to the high number of scenario FTRT simulations which are possibly required, care must be taken to tune the size and resolution of the computational meshes. Computational constraints in the HPC facilities are considered for setting up appropriate meshes, following the three-level grid setup built up within the FTRT workflow.

While results from the FTRT simulations are fed into the system, resulting probabilities for selected tsunami metrics (e.g. maximum tsunami inundation height, tsunami wave heights) are evaluated in order to quantify the tsunami uncertainties for the event. As shown in the workflow diagram, this is achieved by convolving the FTRT outputs with the conditional source probabilities.

The PTF workflow contains functionality for rapid post assessment of the tsunami probability distributions. For this purpose, a set of predefined POIs are readily available for the post assessment. The POIs can be points within the computational domain close to the coast or can coincide with real time observation points such as Deep Ocean Alert system for Tsunami (DART) buoys (https://nctr.pmel.noaa.gov/Dart/). The latter allow us to compare the simulations with real signals of the tsunami. https://nctr.pmel.noaa.gov/Dart/

A global set of coastal POIs are provided for rapidly estimating possible ranges of the maximum tsunami inundation height. Since the employed model for estimating the MIH's, the amplification model, is approximate, the amplification factors also contain information about the uncertainty of the MIHs. These uncertainties, related to the amplification factor method, are also included into the uncertainty estimation. As for the prior source probabilities, the amplification factors for the Mediterranean [14] are based on the TSUMAPS-NEAM project, while the remaining amplification factors [13] are based on the first global probabilistic tsunami hazard analysis [16].

Finally, the PTF workflow contains tools for converting the results to Alert Levels (ALs) once the tsunami uncertainty aggregated at coastal POIs are available. The ALs are determined by combining the tsunami-inundation-height probability with predefined conversion rules (provided by the decision makers) at all the POIs.

\section{FUTURE WORK AND CONCLUDING REMARKS}

We are now able to simulate tsunami propagation multiple times faster than the physical propagation time of a tsunami. Also, a prototype using FTRT for estimating the tsunami uncertainty has been developed and been put to use for in the Mediterranean region. In this paper, we outline two new workflows for future FTRT and PTF. These workflows are developed in the ChEESE project and will be launched in a tier-0 PRACE HPC system. This will allow much faster simulations than previously. An important part of the assessment will be to monitor how launching the simulations in such HPC systems can raise the bar for utilizing HPC in tsunami early warning and rapid post disaster assessments.

While our goal is that the workflows and simulations tools developed in the ChEESE project will be included in operational TEWS after further development sometime in the future, it is expected that the present workflows will provide 
readily available post event assessment tools. The launch of the workflows in a relevant tier-0 HPC environment combined using Tsunami-HySEA as the core computational engine is a necessary condition for acquiring this successfully within minutes to hours just after an event has taken place. This has a typical application foreseen and is already being implemented in the ARISTOTLE Project (http://aristotle.ingv.it/), which provides rapid post-event assessment to the European Civil Protection Mechanism. ARISTOTLE expert advice provided to the Emergency Response Coordination Centre would be greatly supported by both FTRT and PTF tools.

\section{ACKNOWLEDGMENTS}

The authors would like to thank among others R. Tonini, B. Brizuela, M. C. Lorenzino, F. Romano, S. Glimsdal, J.M. González-Vida, and M.J. Castro and other colleagues at INGV, NGI, and Univ. Málaga for their work related to this paper.

The maps in Fig. 2 are generated using the Generic Mapping Tools software [17].

Real seismic waveforms in Fig. 3 are courtesy of the Aristotle University of Thessaloniki Seismological Network, Greece, [18] and the Centroid Moment Tensor solutions for historical earthquakes in the region are obtained from https://www.globalcmt.org/ [19]-[20].

\section{REFERENCES}

[1] NCEI, "NGDC/WDS Global Historical Tsunami Database," https://www.ngdc.noaa.gov/hazard/tsu_db.shtml, August 2019. https://doi.org/10.7289/V5PN93H7

[2] E. A. Okal, "The quest for wisdom: lessons from 17 tsunamis," 20042014. Phil. Trans. R. Soc. A, vol. 373(2053), 20140370, 2015. https://doi.org/10.1098/rsta.2014.0370

[3] F. Løvholt, S. Fraser, M. Salgado-Galvez et al., "Global trends in advancing tsunami science for improved hazard and risk understanding", Contributing Paper to GAR19, June 2019. https://www.unisdr.org/we/inform/publications/65806

[4] E. Bernard and V. Titov, "Evolution of tsunami warning systems and products," Phil. Trans. R. Soc. A, vol. 373(2053), 20140371, 2015. https://doi.org/10.1098/rsta.2014.0371

[5] A. Lomax, A. Michelini, and A. Piatanesi, "An energy-duration procedure for rapid determination of earthquake magnitude and tsunamigenic potential," Geophys. J. Int., vol. 170(3), pp 1195-1209, 2007. https://doi.org/10.1111/j.1365-246X.2007.03469.X

[6] J. Behrens and F. Dias, "New computational methods in tsunami science", Phil. Trans. R. Soc. A, vol. 373(2053), 20140382, 2015. https://doi.org/10.1098/rsta.2014.0382

[7] J. Macías, M. J. Castro, S. Ortega, C. Escalante, and J. M. GonzálezVida, "Performance benchmarking of Tsunami-HySEA model for NTHMP’s inundation mapping activities", Pure Appl. Geophys., vol.
174(8), pp 3147-3183, 2017. https://doi.org/10.1007/s00024-017$\underline{1583-1}$

[8] Y. Okada, "Surface deformation due to shear and tensile faults in a halfspace", Bull. Seism. Soc. Am., vol. 75(4), pp 1135-1154, 1985.

[9] K. Kajiura. "The leading wave of a tsunami", Bull. Earthq. Res. Inst., 41:535-571, 1963.

[10] Scala, A., Lorito, S., Romano, F., Murphy, S., Selva, J., Basili, R., A. Babeyko, A. Herrero, A. Hoehner, F. Løvholt, F.E. Maesano, P. Perfetti, M.M. Tiberti, R. Tonini, M. Volpe, G. Davies, G. Festa, W. Power, A. Piatanesi, and A. Cirella (2019). "Effect of shallow slip amplification uncertainty on probabilistic tsunami hazard analysis in subduction zones: Use of long-term balanced stochastic slip models", Pure and Applied Geophysics, 1-24.

[11] A. Grezio, A. Babeyko, M.A. Baptista, et al., "Probabilistic Tsunami Hazard Analysis: Multiple sources and global applications", Rev.

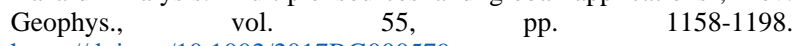
https://doi.org/10.1002/2017RG000579

[12] F.O. Strasser, M. C. Arango, and J.J. Bommer, "Scaling of the source dimensions of interface and intraslab subduction-zone earthquakes with moment magnitude", Seism. Res. Lett. Vol. 81(6), pp 941-950, 2010. https://doi.org/10.1785/gssrl.81.6.941

[13] F. Løvholt, S. Glimsdal, C. B. Harbitz, N. Zamora, F. Nadim, P. Peduzzi, H. Dao, and H. Smebye, "Tsunami hazard and exposure on the global scale", Earth-Sci. Rev., 110(1-4), pp. 58-73, 2012. https://doi.org/10.1016/j.earscirev.2011.10.002

[14] S. Glimsdal, F. Løvholt, C.B. Harbitz, et al., "A new approximate method for quantifying tsunami maximum inundation height probability", Pure Appl. Geophys., vol. 176(7), pp 3227-3246, 2019. https://doi.org/10.1007/s00024-019-02091-w

[15] F. Bernardi, A. Lomax, A. Michelini, V. Lauciani, A. Piatanesi, and S. Lorito, "Appraising the EARLY-EST earthquake monitoring system for tsunami alerting at the Italian Candidate Tsunami Service Provider", Nat. Hazards Earth Syst. Sci., vol. 15, pp. 2019-2036, 2015. https://dx.doi.org/10.5194/nhess-15-2019-2015

[16] G. Davies, J. Griffin, F. Løvholt, et al., "A global probabilistic tsunami hazard assessment from earthquake sources," Geol. Soc. London, Special Publ., vol. 456(1), $\quad$ pp 219-244, 2018. https://doi.org/10.1144/SP456.5

[17] P. Wessel and W. H. F. Smith, " New version of the Generic Mapping Tools released", EOS Trans. AGU, vol 76 (33), pp. 329-329, 1995. https://doi.org/10.1029/95EO00198

[18] Aristotle University of Thessaloniki Seismological Network, Permanent Regional Seismological Network operated by the Aristotle University of Thessaloniki. International Federation of Digital Seismograph Networks. Dataset/Seismic Network, 1981. https://dx.doi.org/10.7914/SN/HT

[19] A. M. Dziewonski, T. A. Chou, and J. H. Woodhouse, " Determination of earthquake source parameters from waveform data for studies of global and regional seismicity", J. Geophys. Res., vol. 86, pp. 28252852, 1981. https://doi.org/10.1029/JB086iB04p02825

[20] G. Ekström, M. Nettles, and A. M. Dziewonski, "The global CMT project 2004-2010: Centroid-moment tensors for 13,017 earthquakes", Phys. Earth Planet. Inter., vol. 200-201, pp. 1-9, 2012. https://doi.org/10.1016/j.pepi.2012.04.002 